\title{
Efficient Feedback via Subspace-Based Channel Quantization for Distributed Cooperative Antenna Systems with Temporally Correlated Channels
}

\author{
Jee Hyun Kim, ${ }^{1}$ Wolfgang Zirwas, ${ }^{1}$ and Martin Haardt ${ }^{2}$ \\ ${ }^{1}$ Nokia Siemens Networks GmbH \& Co. KG, St.-Martin-Strasse 76, 81541 Munich, Germany \\ ${ }^{2}$ Communications Research Laboratory, Ilmenau University of Technology, P.O. Box 100565, 98684 Ilmenau, Germany
}

Correspondence should be addressed to Jee Hyun Kim, jee.kim@nsn.com

Received 15 June 2007; Revised 28 September 2007; Accepted 23 November 2007

Recommended by Ana Pérez-Neira

It is one of the biggest challenges of distributed cooperative antenna (COOPA) systems to provide base stations (BSs) with downlink channel information for transmit filtering (precoding). In this paper, we propose a novel feedback scheme via a subspace-based channel quantization method. The proposed scheme adopts the chordal distance as a channel quantizer criterion so as to capture channel characteristics represented by subspaces spanned by the channel matrix. We also propose a combined feedback scheme which is based on the hierarchical codebook construction method in an effort to reduce the feedback overhead by exploiting the temporal correlation of the channel. The proposed methods are tested for distributed COOPA systems in terms of simulations. Simulation results show that the proposed subspace-based channel quantization method outperforms the analog pilot retransmission method, and the combined feedback scheme performs as well as the permanent full-feedback scheme with a much smaller amount of uplink resources.

Copyright (c) 2008 Jee Hyun Kim et al. This is an open access article distributed under the Creative Commons Attribution License, which permits unrestricted use, distribution, and reproduction in any medium, provided the original work is properly cited.

\section{INTRODUCTION}

Cooperative antenna (COOPA) systems have recently become a hot research topic, as they promise significantly higher spectral efficiency than conventional cellular systems [1]. COOPA systems are also termed coordinated network systems in some references [2-5]. The gain is acquired by adopting intercell interference (ICI) cancelation schemes, for example, joint transmission/joint detection (JT/JD) algorithms. An improvement factor of more than 5 in spectral efficiency is observed for COOPAsystems with an antenna arrangement of 4 transmit antennas per base station and 4 receive antennas per user, compared with uncoordinated cellular systems [2]. In COOPA systems, several adjacent base stations (BSs) are cooperating so as to support multiple mobile stations (MSs) which are located in the corresponding cooperative area (CA). Therefore, COOPA systems can be regarded as a multiuser multiple-input multiple-output (MU-MIMO) system, in which multiple transmit antennas at the BS, which are conventionally considered to be located in one BS, are spread over several BSs. This distributed na- ture, which is attributed to the fact that several geographically distributed BSs are used as transmit antennas, leads to full macro-diversity gains. Moreover, COOPA systems have advantageous features compared with conventional cellular systems, for example, increased degrees of freedom, better ICI cancelation performance, the rank enhancement effect of the channel matrix, and so forth, [1]. In addition, JT/JD algorithms of COOPA systems calculate a common weighting matrix for all BSs, cancel ICI, and allow the system to serve multiple MSs at the same time and frequency resource. This leads to a real-frequency reuse equal or close to 1 .

COOPA systems are based on the cooperation between multiple distributed BSs. This means that COOPA systems need a fast and efficient backbone network as well as the central unit (CU) which manages the cooperation amongst associated BSs. The CU renders the overall network structure more complex by adding one more layer in the hierarchy, and eventually increases the costs. In [6], distributed organization methods have been suggested to address this problem.

One of the main challenges of the distributed COOPA system is channel estimation for the downlink channel. All 
of the associated BSs in the CA need to know the full channel state information to calculate the corresponding precoding weight matrix. This information is needed to be transferred from MSs to BSs by using uplink resources. As several BSs and several MSs are involved in COOPA systems and each BS and MS may be equipped with multiple antennas, the number of channel state parameters to be fed back is expected to be big. In an effort to reduce the amount of feedback, the analog pilot retransmission method has been suggested and tested in [6], but the throughput of this method reaches only $40 \%$ of that of the ideal case, which requires supplementary feedback schemes [1].

On the other hand, finite rate feedback strategies in MIMO systems have been extensively investigated recently. Beamforming codebook design methods are suggested based on Grassmannian packing [7] and systematic unitary design [8], which guarantee substantial gains with just a small number of feedback bits. A precoding matrix codebook construction method, which is designed to maximize the mutual information, has been developed based on vector quantization (VQ) techniques $[9,10]$. These methods are designed to select a beamforming vector for the MISO case or a precoding matrix for the MIMO case from a set of codes. They are developed for point-to-point MIMO channels, in which the transmitter serves one receiver at a time. In the single-user MIMO (SU-MIMO) case, it is known that even a small number of bits per antenna can be quite beneficial [11]. In the multiuser MIMO (MU-MIMO) case, feedback rate scaling is required to achieve a throughput close to that with perfect feedback information in order to compensate for the interference between users [12]. The analysis in [12] is based on the case when a user selects the precoding matrix by solely looking at its own channel without considering the interference to other users which is caused by adopting that precoding matrix. Hence, a better way to handle interuser interference needs to be addressed.

In this paper, we propose a subspace-based channel quantization method which guarantees a much higher performance than the analog pilot retransmission method. We also propose an iterative codebook design algorithm which converges to a locally optimum codebook. Furthermore, as a feedback reduction scheme, we propose a hierarchical codebook design method. The proposed schemes can be used for cellular MU-MIMO systems as well, which involve one BS for downlink data transmission.

\section{Notation}

Vectors and matrices are denoted by lower case bold and capital bold letters, respectively. $(\cdot)^{\mathcal{T}}$ and $(\cdot)^{\mathscr{H}}$ denote transpose and Hermitian transpose, respectively. The inner product between two vectors is defined as $\langle\mathbf{u}, \mathbf{v}\rangle=\mathbf{u}^{\mathscr{H}} \mathbf{v} \cdot \operatorname{tr}(\cdot)$ denotes the trace of a matrix. $|\cdot|,\|\cdot\|_{2}$, and $\|\cdot\|_{F}$ denote the magnitude of a scalar, the two-norm of a vector or a matrix, and the Frobenius norm of a matrix, respectively. The covariance matrix of the vector process $\mathbf{x}$ is denoted by $\mathbf{R}_{\mathbf{x}}=\mathbb{E}\left[\mathbf{x x}^{\mathscr{H}}\right]$, where $\mathbb{E}[\cdot]$ is used for expectation. $\mathbf{I}_{N}$ is the $N \times N$ identity matrix and $\mathbf{0}_{M \times N}$ stands for an all-zero matrix of size $M \times N$. $\mathbf{I}_{M \times N}$ is defined as $\mathbf{I}_{M \times N}:=\left[\begin{array}{c}\mathbf{I}_{N} \\ \mathbf{0}_{(M-N) \times N}\end{array}\right]$ for $M>N .[\mathbf{A}]_{i, j}$ stands for the $(i, j)$ th entry of a matrix A. $|\&|$ is the cardinality of a set $\&$.

\section{SYSTEM MODEL AND MOTIVATION FOR CHANNEL QUANTIZATION METHOD}

We consider a precoded MU-MIMO system in which a group of BSs transmits data to multiple MSs simultaneously. Each of $N_{\mathrm{BS}}$ BSs and each of $N_{\mathrm{MS}}$ MSs have $N_{t}$ and $N_{r}$ antennas, respectively. The data symbol block, $\mathbf{s}=\left[s_{1}, \ldots, s_{N_{t r}}\right]^{\mathcal{T}}$ with $N_{t r}=N_{\mathrm{MS}} N_{r}$, is precoded by an $N_{t t} \times N_{t r}$ matrix $\mathbf{W}$ with $N_{t t}=N_{\mathrm{BS}} N_{t}$, in case that the number of data streams for each user $n_{s}\left(n_{s} \leq N_{r}\right)$ is $N_{r}$. Here, the first $N_{r}$ data symbols are intended for the first user, the next $N_{r}$ symbols for the second user, and so on. When denoting $i_{\mathrm{BS}} / i_{\mathrm{MS}}$ as the BS/MS index and $i_{t} / i_{r}$ as the transmit/receive antenna index, respectively, we can denote $h_{i, j}$, where $i=N_{r}\left(i_{\mathrm{MS}}-1\right)+i_{r}, j=N_{t}\left(i_{\mathrm{BS}}-1\right)+$ $i_{t}$ as the channel coefficient between the $i_{r}$ th receive antenna of the $i_{\mathrm{MS}}$ th MS and the $i_{t}$ th transmit antenna of the $i_{\mathrm{BS}}$ th BS. The $N_{t r} N_{t t}$ channel coefficients can be expressed as the $N_{t r} \times$ $N_{t t}$ channel matrix $\mathbf{H}$ with $[\mathbf{H}]_{i, j}=h_{i, j}$. The received signals on $N_{t r}$ receive antennas which are collected in the vector $\mathbf{y}$ can be formulated as

$$
\mathbf{y}=\mathrm{HWs}+\mathbf{n},
$$

where $\mathbf{n}$ is additive white Gaussian noise (AWGN). The signal model appears to be very similar to that of the singleuser MIMO case at a first glance, but the difference lies in the fact that the channel matrix in our case contains elements belonging to multiple BSs and multiple MSs.

There are several available techniques developed for downlink transmit filtering in MU-MIMO systems. Linear precoding techniques (e.g., transmit matched filter (TxMF), transmit zero-forcing filter (TxZF), and transmit Wiener filter (TxWF) have an advantage in terms of computational complexity [13]. Nonlinear techniques (e.g., TomlinsonHarashima precoding (THP)) have a higher-computational complexity but can usually provide a better performance than linear techniques $[14,15]$. Some linear techniques (e.g., block diagonalization (BD) and successive minimum mean squared error precoding (SMMSE)) are developed for the case in which there are multiple antennas at each receiver. The BD algorithm is designed to eliminate multiuser interference (MUI) [16]. BD outperforms the TxZF and asymptotically approaches the sum capacity of the channel at high SNR. SMMSE performs better than some nonlinear techniques (e.g., successive optimization (SO) THP and MMSE THP) with a relatively low-computational complexity [17]. In our case, we adopt the TxZF which completely suppresses the interference at the receiver [13] as follows:

$$
\begin{aligned}
\{\mathbf{W}, g\} & =\arg \min _{\{\mathbf{W}, g\}}|g|^{2} \operatorname{tr}\left(\mathbf{R}_{\mathbf{n}}\right) \\
\text { s.t.: } g \mathbf{H W} & =\mathbf{I}_{N_{t r}}, \quad \operatorname{tr}\left(\mathbf{W R}_{\mathbf{s}} \mathbf{W}^{\mathcal{H}}\right)=P_{t x}
\end{aligned}
$$

where $P_{t x}, \mathbf{R}_{\mathbf{n}}$, and $\mathbf{R}_{\mathbf{s}}$ are the maximum transmit power, the covariance matrix of the noise, and the covariance matrix of the data symbol, respectively. The TxZF strategy, while generally suboptimal, is known to achieve the same asymptotic 
sum capacity as that of dirty paper coding (DPC) which is the optimal (channel capacity achieving) method, as the number of users goes to infinity [18]. The transmit precoding matrix W which satisfies the design criteria (2) takes the following form:

$$
\begin{aligned}
\mathbf{W} & =g^{-1} \mathbf{H}^{\mathcal{H}}\left(\mathbf{H} \mathbf{H}^{\mathcal{H}}\right)^{-1}, \\
\text { where } g & =\sqrt{\frac{\operatorname{tr}\left(\left(\mathbf{H} \mathbf{H}^{\mathcal{H}}\right)^{-1} \mathbf{R}_{\mathbf{s}}\right)}{P_{\text {tr }}}} .
\end{aligned}
$$

The challenge here is that BSs should know the downlink channel matrix $\mathbf{H}$ so as to construct the precoding matrix $\mathbf{W}$. The analog pilot retransmission method has been proposed as a way of transferring channel state information to the BS [6]. As shown in [6], the analog pilot retransmission method is vulnerable to noise enhancement effects and this weakness of the analog method brings about a significant performance degradation, even though it is efficient in terms of required resources. As a way of combating noise, a digital method can be used instead of the analog method. A digital method implies that MSs measure the downlink channel and encode this information into a digital code and send it back to the BSs after performing appropriate digital signal processing (modulation, spreading, repetition, or channel coding, etc.) to guarantee robust data transmission.

As it is explained in the previous section, most of the finite rate feedback strategies in MIMO systems are designed for the single user case, focusing on the selection and construction of the precoding matrix codebook. If this strategy is directly applied to the multiuser case, the performance will be degraded since the user is supposed to select the precoding matrix which is suitable in terms of its criterion (e.g., maximizing the mutual information or SNR), which may cause a severe interference to other users. Here, we propose to quantize the channel from the MS side instead of quantizing the precoding matrix. Both methods are similar from the signal processing perspective in the sense that both schemes compress the information in a matrix, while the channel quantization method is better positioned to cope with interuser interferences. The BSs, after receiving feedback messages from the MSs, can now build a precoding matrix with interuser interferences taken into account, since the transmitters have the whole channel state information, albeit it is not perfect due to the limited feedback.

One way of quantizing the channel matrix is to view the channel matrix as a set of complex matrices, and to quantize every individual matrix by looking up a predefined codebook. As explained above, the overall channel matrix is an $N_{\mathrm{MS}} N_{r} \times N_{\mathrm{BS}} N_{t}$ matrix, and is composed of the channel matrices for each user, which are of size $N_{r} \times N_{\mathrm{BS}} N_{t}$. Equation (4) depicts this relationship as follows:

$$
\mathbf{H}=\left[\mathbf{H}_{1}, \ldots, \mathbf{H}_{j}, \ldots, \mathbf{H}_{N_{\mathrm{MS}}}\right]^{\mathcal{T}}, \quad j: \text { user index. }
$$

Here, $\mathbf{H}_{j}$ is the transpose of the channel matrix for user $j$, which is an $N_{\mathrm{BS}} N_{t} \times N_{r}$ matrix. If we allocate $n_{\mathrm{CB}}$ bits for the codebook, we need $n_{\mathrm{CB}} N_{\mathrm{MS}}$ bits in total for every subcarrier. This method is suitable for the limited feedback in terms of required feedback bits, and the conventional vector quantization (VQ) method can be applied with some modifications.

The system model is depicted in Figure 1. The $N_{\text {BS }}$ BSs need overall downlink channel state information $\mathbf{H}$ for the calculation of the precoding matrix $\mathbf{W}$ so as to form multiple spatial beams which enable independent and decoupled data streams for $N_{\text {Ms }}$ users. The individual user $j$ estimates its portion of the channel $\mathbf{H}_{j}$ and quantizes it by finding the best candidate from the predefined set of codes $\mathbf{C}_{i}$. The index of the chosen code $i_{j}$ is sent back to the BSs through the limited feedback channel. The BSs reconstruct the channel matrix $\hat{\mathbf{H}}$ by looking up the codebook, which is shared by transmitters and receivers. This reconstructed channel matrix is used for the calculation of the precoding matrix $\mathbf{W}$. We should note that in this case all of the $N_{\mathrm{BS}}$-associated BSs have the same channel matrix, as long as the feedback messages are received without errors. In case of the analog pilot retransmission method, the individual BS has its own version of the channel matrix, which is in general different from each other due to the nature of the analog transmission scheme, and this entails a significant performance degradation $[1,6]$. The principles of the analog pilot retransmission method can be found in [1, Section 10.3.3.1].

\section{SUBSPACE-BASED CHANNEL QUANTIZATION METHOD}

As proposed in the previous section, MS $j$ is supposed to quantize its channel matrix $\mathbf{H}_{j}$. We view $\mathbf{H}_{j}$ not just as a complex matrix but as a subspace which is spanned by its columns. We perform a singular value decomposition (SVD) to extract the unitary matrix $\mathbf{U}_{j}$ which includes the basis vectors $\mathbf{U}_{j}^{(S)}$ spanning the column space of $\mathbf{H}_{j}\left(\mathbf{H}_{j}: N_{t t} \times N_{r}, \mathbf{U}_{j}\right.$ : $\left.N_{t t} \times N_{t t}, \mathbf{U}_{j}^{(S)}: N_{t t} \times N_{r}\right)$. Here, the superscripts (S) and (0) are used to denote a basis for the signal subspace and the null space, respectively,

$$
\mathbf{H}_{j}=\mathbf{U}_{j} \boldsymbol{\Sigma}_{j} \mathbf{V}_{j}^{\mathcal{H}}, \quad \mathbf{U}_{j}=\left[\begin{array}{ll}
\mathbf{U}_{j}^{(S)} & \mathbf{U}_{j}^{(0)}
\end{array}\right] .
$$

The channel quantizer uses the chordal distance as a distance metric, since we should measure the distance between subspaces. There are other subspace distance metrics [19], but the chordal distance is the one which leads us to an analytic solution when designing the codebook [20]. The chordal distance is defined as

$$
d_{c}\left(\mathbf{T}_{i}, \mathbf{T}_{j}\right)=\frac{1}{\sqrt{2}}\left\|\mathbf{T}_{i} \mathbf{T}_{i}^{\mathscr{H}}-\mathbf{T}_{j} \mathbf{T}_{j}^{\mathcal{H}}\right\|_{F}
$$

for matrices $\mathbf{T}_{i}, \mathbf{T}_{j}$ which have orthonormal columns.

The quantized version of the column space basis vectors $\mathbf{U}_{j}^{(S)}$ is chosen to be the code which has the minimum chordal distance from it. Thus, the subspace quantization process can be written as

$$
\widehat{\mathbf{U}}_{j}^{(S)}=\mathcal{Q}\left(\mathbf{U}_{j}^{(S)}\right)=\arg \min _{\mathbf{C}_{i} \in \mathcal{C}} d_{c}\left(\mathbf{U}_{j}^{(S)}, \mathbf{C}_{i}\right),
$$

where $\mathcal{C}$ is the codebook of size $N\left(N=2^{n_{\mathrm{CB}}}\right)$ which has the code $\mathbf{C}_{i} \in \mathbb{C}^{N_{t t} \times N_{r}}$ as its elements. Here, $\mathbf{C}_{i}$ has unitary 


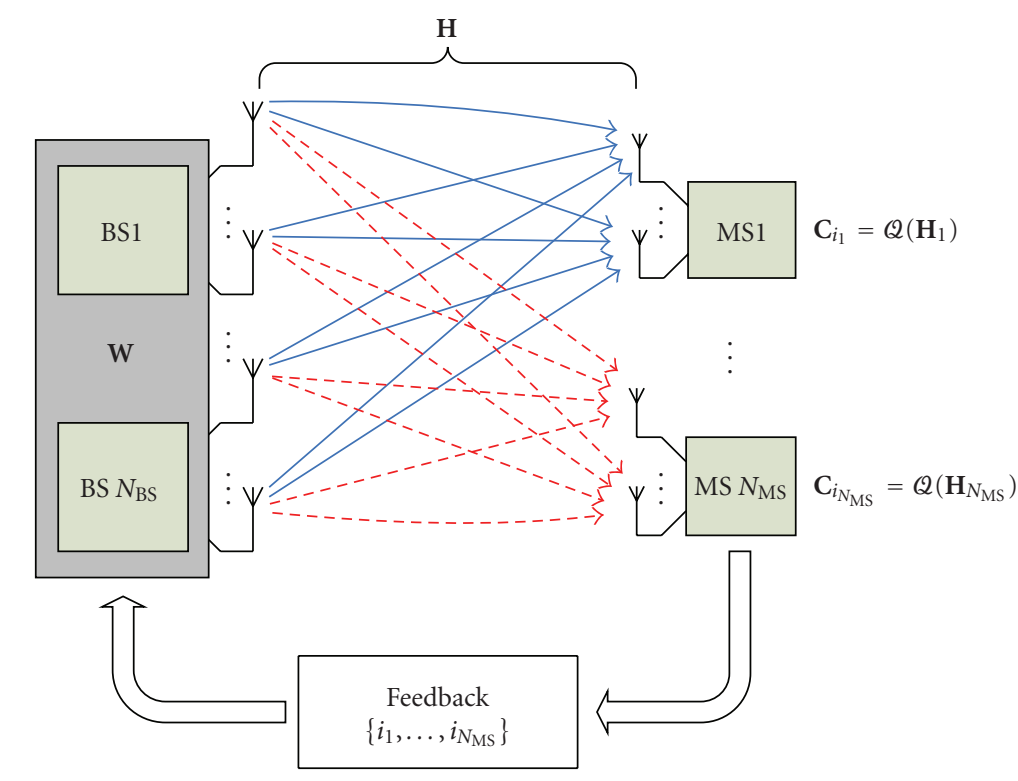

$$
\hat{\mathbf{H}}=\left[\begin{array}{lll}
\mathbf{C}_{i_{1}} & \cdots & \mathbf{C}_{i_{N_{\mathrm{MS}}}}
\end{array}\right]^{\mathcal{T}}
$$

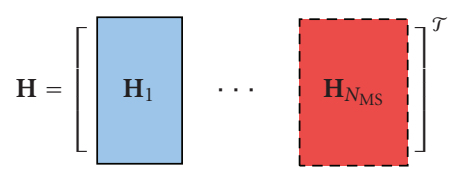

FIgURE 1: COOPA system downlink with $N_{\mathrm{BS}}$ cooperating base stations and $N_{\mathrm{MS}}$ mobile stations.

columns $\left(\mathbf{C}_{i}^{\mathscr{H}} \mathbf{C}_{i}=\mathbf{I}_{N_{r}}\right)$, hence the code represents only the column space of $\mathbf{H}_{j}$. No channel magnitude information is fed back to the transmitter, since extensive simulation results show that extra magnitude information does not improve the system performance, compared with the case in which only the code index is provided to the transmitters when the link strengths (large-scale fading due to path loss and shadowing) are assumed to be provided at the BSs. In the case that channel magnitude information is to be fed back, the quantized version of the channel at the transmitter which takes this into account can be formulated as

$$
\widehat{\mathbf{H}}_{j}=\widehat{\mathbf{U}}_{j}^{(S)} \boldsymbol{\Sigma}_{j}^{(S)}
$$

where $\boldsymbol{\Sigma}_{j}^{(S)} \in \mathbb{R}_{+}^{N_{r} \times N_{r}}$ is a diagonal matrix which is composed of $N_{r} \times N_{r}$ elements in the upper left-hand corner of $\boldsymbol{\Sigma}_{j}$. The diagonal elements of $\Sigma_{j}^{(S)}$ constitute the channel magnitude information, which can be regarded as a refinement of the link strengths which are already available at the BSs. This channel quantization model (8) can provide a better view of the channel, as it considers not only the channel directional information, but also the channel magnitude information (link strength refinement information). However, the simulation results show that this extra information does not enhance the system performance in terms of SINR, compared with the case in which only the channel directional information is provided. Some of the simulation results can be found in Section 6. It is known that the performance can be improved by providing the transmitter with the channel quality information (e.g., SINR) in addition to the directional information (the column space basis vectors), when a multiantenna downlink system carrying more users than transmit antennas is considered [21]. In our case, the multiuser diversity gain is not considered at the moment, so we focus on the directional information of the channel. The bottom line is that the MS needs to quantize the column space of $\mathbf{H}_{j}$ only. The channel magnitude information contained in $\boldsymbol{\Sigma}_{j}$ is, therefore, not required. In this case, the MS is supposed to send only $n_{\mathrm{CB}}$ bits of feedback. The channel quantization formula can be simplified as

$$
\hat{\mathbf{H}}_{j}^{d}=\hat{\mathbf{U}}_{j}^{(S)}=\arg \min _{\mathbf{C}_{i} \in \mathcal{C}} d_{c}\left(\mathbf{U}_{j}^{(S)}, \mathbf{C}_{i}\right) .
$$

The superscript $d$ implies that the channel is quantized in terms of the direction, with its magnitude information ignored.

The subspace-based channel quantization method works as follows. MS $j$ finds the code $\mathrm{C}_{i}$ which provides the minimum chordal distance with $\mathbf{U}_{j}^{(S)}$. Then, it sends back an $n_{\mathrm{CB}}$ bit code index to all associated BSs. The reconstructed downlink channel matrix at the BSs is as follows:

$$
\hat{\mathbf{H}}=\left[\hat{\mathbf{H}}_{1}^{d}, \ldots, \hat{\mathbf{H}}_{j}^{d}, \ldots, \hat{\mathbf{H}}_{N_{\mathrm{MS}}}^{d}\right]^{\mathcal{T}}, \quad j: \text { user index. }
$$

Finally, the BSs calculate the TxZF precoding matrix $\mathbf{W}$ by using the reconstructed channel matrix $\hat{\mathbf{H}}$ as follows:

$$
\mathbf{W}=g^{-1} \hat{\mathbf{H}}^{\mathcal{H}}\left(\hat{\mathbf{H}} \hat{\mathbf{H}}^{\mathcal{H}}\right)^{-1},
$$


where $g$ is the normalization factor imposed by the transmit power constraint (3). (Actually, it is not a true zero-forcing precoding matrix in the strict sense, since the channel magnitude information is not considered. In this paper, we use the term TxZF interchangeably for this particular case, assuming that readers are not to be confused.)

It is worth noticing that the channel quantization criterion (9) can be expressed as

$$
\widehat{\mathbf{h}}_{j}=\arg \max _{\mathbf{c}_{i} \in \mathcal{C}}\left|\left\langle\mathbf{v}_{j}, \mathbf{c}_{i}\right\rangle\right|, \quad \text { where } \mathbf{v}_{j}=\frac{\mathbf{h}_{j}}{\left\|\mathbf{h}_{j}\right\|_{2}},
$$

for the MISO case where the MS is equipped with one antenna. In this case, the task of quantizing the channel boils down to that of quantizing the channel vector, instead of the channel matrix. It basically selects the code of which the direction is closely aligned with the direction of the channel. Here, the channel to be quantized, the directional information of the channel, and the corresponding code are all vectors of the same size $\left(\mathbf{h}_{j}, \mathbf{v}_{j}, \mathbf{c}_{j} \in \mathbb{C}^{N_{t t} \times 1}\right)$. For the proof of this formula, please refer to the appendix.

\section{CODEBOOK CONSTRUCTION BASED ON MODIFIED LBG VQ ALGORITHM}

The Grassmannian subspace packing is optimal in terms of quantization for the uncorrelated Rayleigh fading channel [7]. The Grassmannian space $g(m, n)$ is the set of all $n$ dimensional subspaces of the space $\mathbb{C}^{m}$, and the Grassmannian subspace packing problem is the problem of finding the best packing of $N n$-dimensional subspaces in $\mathbb{C}^{m}$. The best packing means that $N$ points in $\mathscr{g}(m, n)$ are maximally spaced such that the minimal distance between any two of the subspaces is as large as possible.

In our case, the Linde, Buzo, and Gray (LBG) vector quantization (VQ) algorithm [22] is used to construct the codebook $\mathcal{C}$. The LBG VQ algorithm is an iterative algorithm based on the Lloyd's algorithm which is known to provide an alternative systematic approach for the Grassmannian subspace packing problem [20]. We in this paper acquire the codebook through the iterative algorithm described in [20]. The main difference of the proposed method is attributed to the fact that the codebooks in [20] are precoder codebooks, while the codebooks to be constructed here are channel quantizer codebooks. The proposed algorithm aims at finding a tradeoff between good quantization properties and the Grassmannian subspace packing requirements by adopting the minimum chordal distance of the codebook as a decision criterion for iterations.

\subsection{Design issue}

The LBG-VQ-based codebook $\mathcal{C}$ design problem can be stated as follows. For a given source vector, a given distortion measure, a given codebook evaluation measure, and given the size of the codebook, find a codebook and a partition which result in maximizing the minimum chordal distance of the codebook. (The partition of the space is defined as the set of all encoding regions.) In other words, we want to find maximally spaced $N$ points in $\mathcal{G}\left(N_{t t}, N_{r}\right)$ with given channel realization samples.

Suppose that we have a training sequence $\mathcal{T}$ to capture the statistical properties of the column space basis vectors $\mathbf{U}_{j}^{(S)}$ of size $N_{t t} \times N_{r}$ :

$$
\mathcal{T}=\left\{\mathbf{X}_{1}, \mathbf{X}_{2}, \ldots, \mathbf{X}_{M}\right\}
$$

where $\mathbf{X}_{m} \in \mathbb{C}^{N_{t t} \times N_{r}}$ is a sample of $\mathbf{U}_{j}^{(S)}$ which can be obtained by taking an SVD of the channel matrix $\mathbf{H}_{j}$. The codebook can be represented as follows:

$$
\mathcal{C}=\left\{\mathbf{C}_{1}, \mathbf{C}_{2}, \ldots, \mathbf{C}_{N}\right\}
$$

The individual code is of the same size as a training matrix $\left(\mathbf{C}_{n} \in \mathbb{C}^{N_{t t} \times N_{r}}\right)$. Let $\mathcal{R}_{n}$ be the encoding region associated with the code $\mathbf{C}_{n}$ and let

$$
\mathcal{P}=\left\{\mathcal{R}_{1}, \mathcal{R}_{2}, \ldots, \mathcal{R}_{N}\right\}
$$

denote the partition of the space. (The encoding region is called a Voronoi cell in some publications.) If the source matrix $\mathbf{X}_{m}$ belongs to the encoding region $\mathcal{R}_{n}$, then it is quantized to $\mathbf{C}_{n}$ as follows:

$$
\mathcal{Q}\left(\mathbf{X}_{m}\right)=\mathbf{C}_{n}, \quad \text { if } \mathbf{X}_{m} \in \mathcal{R}_{n} .
$$

Our aim is to find a codebook of which the minimum chordal distance is maximized. There are several subspace distance metrics, for example, the Fubini-Study distance, the projection two-norm distance, and the chordal distance metrics. It has been shown that the chordal distance is the only distance measure which makes the iterative algorithm feasible [20]. The minimum chordal distance of the codebook is given by

$$
d_{c, \min }(\mathcal{C}):=\min d_{c}\left(\mathbf{C}_{i}, \mathbf{C}_{j}\right), \quad \text { for } \mathbf{C}_{i}, \mathbf{C}_{j} \in \mathcal{C}, \forall i \neq j .
$$

The design problem can be stated as follows. Given $\mathcal{T}$ and $N$, find $\mathcal{C}$ and $\mathcal{P}$ such that $d_{c, \min }(\mathcal{C})$ is maximized:

$$
\mathcal{C}_{\mathrm{opt}}=\arg \max _{\mathcal{C}} d_{c, \min }(\mathcal{C})
$$

\subsection{Optimality criteria}

$\mathcal{C}$ and $\mathcal{P}$ must satisfy the following two criteria so as to be a solution to the above-mentioned design problem [22]. We should note that the chordal distance is used as a distance metric.

(i) Nearest neighbor condition:

$$
\mathcal{R}_{n}=\left\{\mathbf{X}: d_{c}\left(\mathbf{X}, \mathbf{C}_{n}\right)<d_{c}\left(\mathbf{X}, \mathbf{C}_{n^{\prime}}\right), \forall n^{\prime} \neq n\right\} .
$$

This condition says that any channel sample $\mathbf{X}$, which is closer to the code $\mathbf{C}_{n}$ than any other codes in the chordal distance sense, should be assigned to the encoding region $\mathcal{R}_{n}$, and be represented by $\mathbf{C}_{n}$.

(ii) Centroid condition:

$$
\mathbf{C}_{n}=\mathbf{U}_{R} \mathbf{I}_{N_{t t} \times N_{r}}
$$


TABle 1: The minimum codebook distances $d_{c, \min }(\mathcal{C})$.

\begin{tabular}{lccc}
\hline$\left(N_{t t}, N_{r}\right)$ & $n_{\mathrm{CB}}$ & mLBG VQ & Grassmann \\
\hline$(2,1)$ & 3 & 0.3895 & 0.3820 \\
$(3,1)$ & 3 & 0.5706 & 0.5429 \\
& 4 & 0.4882 & 0.4167 \\
\hline
\end{tabular}

where $\mathbf{U}_{R}$ is an eigenvector matrix of the sample covariance matrix $\mathbf{R}$ which is defined as

$$
\mathbf{R}:=\frac{1}{N_{\mathcal{R}_{n}}} \sum_{\mathbf{X}_{m} \in \mathcal{R}_{n}} \mathbf{X}_{m} \mathbf{X}_{m}^{\mathcal{H}}, \quad \text { where } N_{\mathcal{R}_{n}}=\left|\mathcal{R}_{n}\right|
$$

provided that eigenvalues in the eigenvalue matrix $\boldsymbol{\Sigma}_{R}$ of $\mathbf{R}=$ $\mathbf{U}_{R} \boldsymbol{\Sigma}_{R} \mathbf{U}_{R}^{\mathscr{H}}$ are sorted in the descending order. This condition means that the code $\mathbf{C}_{n}$ of the encoding region $\mathcal{R}_{n}$ should be the principal eigenvectors of the sample covariance matrix $\mathbf{R}$, meaning the $N_{r}$ eigenvectors of $\mathbf{R}$ corresponding to $N_{r}$ largest eigenvalues. The centroid condition is designed to minimize the average distortion in the encoding region $\mathcal{R}_{n}$, when $\mathbf{C}_{n}^{\mathrm{opt}}$ represents $\mathcal{R}_{n}[20]$.

\subsection{Modified LBG VQ algorithm}

The modified LBG VQ ( $\mathrm{mLBG}$ VQ) design algorithm is an iterative algorithm which finds the solution satisfying the two optimality criteria in Section 4.2. The algorithm requires an initial codebook $\mathcal{C}^{(0)} \cdot \mathcal{C}^{(0)}$ is obtained by the splitting of an initial code, which is the centroid of the entire training sequence, into two codes. The iterative algorithm runs with these two codes as the initial codebook. The final two codes are split into four and the same process is repeated until the desired number of codes, which leads to the minimum chordal distance, is obtained.

The minimum distances of the codebooks are collected in Table 1. A training sequence of the length 50,000 is used for obtaining the codebook. It shows that the codebooks acquired by the modified LBG VQ algorithm have better distance properties than the Grassmannian codebooks listed in [23].

\section{COMBINED CODEBOOK: HIERARCHICAL CODEBOOK DESIGN METHOD}

In this section, we propose a hierarchical codebook design method, which exploits the temporal correlation of the channel, as a way of reducing the feedback overhead. It is known that the wireless channel does not change radically within the coherence time $T_{c}$. Accordingly, the code index would not change so often during this time period, since the code index can be considered as a channel state indicator. On the other hand, we can easily draw the conclusion that the codebook index transition rate over time is dependent on the size of the codebook, which decides the resolution of the channel quantizer. It means that for a given channel, a bigger size

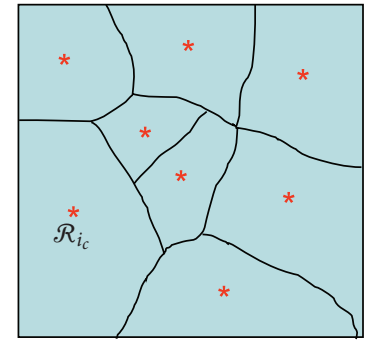

(a) Coarse encoding region

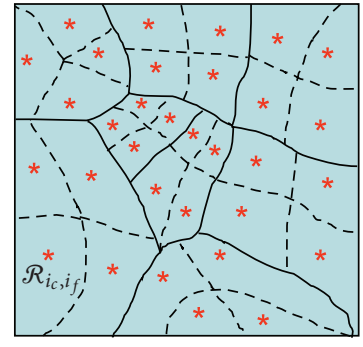

(b) Fine encoding region

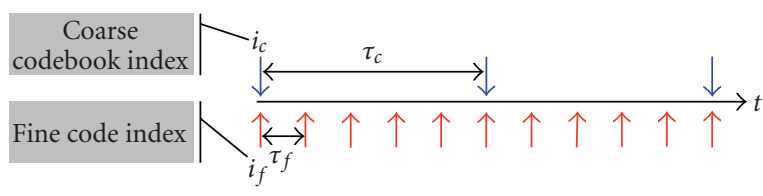

(c)

Figure 2: Coarse/fine encoding regions and feedback time frame.

codebook (fine codebook) has a higher capability of differentiating encoding regions than a smaller size codebook (coarse codebook). The period during which the coarse codebook provides the same code index (let us call this a nontransition period) can be composed of several shorter nontransition periods when the fine codebook is used to quantize the channel.

Thus, if we are able to design a codebook hierarchically so that a codebook has several layers, say two layers, one of which represents coarse encoding regions and another provides fine encoding regions, we can achieve the performance of a fine channel quantizer by using much smaller feedback resources. This can be achieved by organizing the coarse/fine codebook feedback periods in a smart way to take advantage of nontransition periods of the coarse/fine codebook.

The operation scenario of the combined codebook is as follows. As a preparation, we need to design the combined codebook which has a two-layer structure, namely, an $n_{c}$ bit coarse codebook and an $n_{f}$ bit fine codebook. Corresponding feedback periods should be decided, based on the statistical properties of the nontransition time. The $n_{t}=n_{c}+n_{f}$ bit combined codebook, as a whole, is designed to be composed of $2^{n_{c}}$ groups of the fine codes, and each fine codebook group consists of $2^{n_{f}}$ fine codes. The feedback operation works as follows. For every coarse feedback period $\tau_{c}$ of the $n_{c}$ bit coarse codebook, the MS sends the coarse codebook index $i_{c}$ ( $n_{c}$ bit) back to the BSs to indicate the fine codebook group index to which the subsequent fine code indices belong. At the same time, the MS sends the fine code index $i_{f}\left(n_{f}\right.$ bit) to indicate the fine code index of the chosen fine codebook group. This subsequent feedback is done for every fine feedback period $\tau_{f}$ of the $n_{t}$ bit fine codebook. Since that, $n_{c}$ bit feedback is sent back for every $\tau_{c}$ and only $n_{f}$ bit extra feedback is needed for every $\tau_{f}$, we can save uplink resource when compared to the case of sending back $n_{t}$ bit feedback for every time. Interested readers can consult Figure 2 for better understanding. 


\subsection{Hierarchical codebook construction}

The main design problem of hierarchical codebook construction is to divide a coarse encoding region into equally probable fine encoding regions. Here, the term "equally probable" means that the probability that a channel sample falling into a certain encoding region is the same for all candidate encoding regions. Equally probable encoding regions allow us to fix the feedback period for given channel-dependent constraints.

The modified LBG VQ (mLBG VQ) algorithm, which is used for codebook construction, generates the codebook which pertains this property. The resulting codes are maximally spaced codes of which an individual code is designed to provide the minimum mean squared chordal distance between the code and the channel samples in that encoding region. This criterion places finer encoding regions in densely populated areas, and the resulting encoding regions are asymptotically equally probable. This is shown to be true in [24] as well, for codes generated by the Lloyd's algorithmbased codebook construction method.

The design problem of hierarchical codebook construction with an $n_{c}$ bit coarse and an $n_{t}$ bit fine codebook is to divide the given channel space which is a subspace of $\mathbb{C}^{N_{t t} \times N_{r}}$ into $2^{n_{c}}$ equally probable coarse encoding regions and to divide each coarse encoding region into $2^{n_{f}}$ equally probable fine encoding regions. In the end, we want to have $2^{n_{c}}$ groups of codebooks each of which is composed of $2^{n_{f}}$ codes. This can be solved as follows. We first perform the mLBG VQ algorithm to get $N_{c}=2^{n_{c}}$ coarse codes and corresponding coarse encoding regions. These encoding regions are supposed to be equally probable $\left(P_{n}=1 / N_{c}, \forall n \in\left\{1, \ldots, N_{c}\right\}\right)$. Then, we perform the mLBG VQ for channel samples which belong to each coarse encoding region, individually. As a result of $N_{c}$ parallel codebook generation processes for each coarse encoding region, we can acquire $N_{c b}=2^{n_{t}}=2^{n_{c}+n_{f}}$ fine codes with corresponding equally probable fine encoding regions $\left(P_{n}=1 / N_{f}, \forall n \in\left\{1, \ldots, N_{f}\right\}\right)$. Each coarse encoding region consists of $N_{f}=2^{n_{f}}$ fine encoding regions.

The overall codebook $\mathcal{C}$ can be regarded as a set of codebooks $\mathcal{C}_{i_{c}}^{c}$, where $i_{c}$ is the codebook index. Here, we differentiate between the terms code and codebook, in such a way that a code indicates an individual code, whereas a codebook indicates a set of codes. Thus $i_{c}$ indicates not an individual code index, but a codebook index to which a fine code belongs. It means that the codebook $\mathcal{C}_{i_{c}}^{c}$ is constructed based on the $i_{c}$ th coarse encoding region $\mathcal{R}_{i_{c}}$. The elements of $\mathcal{C}_{i_{c}}^{c}$ are fine codes. The overall codebook $\mathcal{C}$ and the $i_{c}$ th fine codebook $\mathcal{C}_{i_{c}}^{c}$ can be expressed as

$$
\begin{gathered}
\mathcal{C}=\left\{\mathcal{C}_{1}^{c}, \mathcal{C}_{2}^{c}, \ldots, \mathcal{C}_{N_{c}}^{c}\right\}=\left\{\mathbf{C}_{1}, \mathbf{C}_{2}, \ldots, \mathbf{C}_{N_{c b}}\right\}, \\
\mathcal{C}_{i_{c}}^{c}=\left\{\mathbf{C}_{1}^{i_{c}}, \mathbf{C}_{2}^{i_{c}}, \ldots, \mathbf{C}_{i_{f}}^{i_{c}}, \ldots, \mathbf{C}_{N_{f}}^{i_{c}}\right\}, \quad \text { for } i_{c} \in\left\{1,2, \ldots, N_{c}\right\},
\end{gathered}
$$

where fine codes are arranged in such an order that the condition $\mathbf{C}_{i_{f}}^{i_{c}}=\mathbf{C}_{\left(i_{c}-1\right) N_{f}+i_{f}} \in \mathbb{C}^{N_{t t} \times N_{r}}$ is satisfied and $i_{f}$ is the fine code index within the coarse encoding region $\mathcal{R}_{i_{c}}$. It becomes clear at this point that the resulting codebook has a hierarchical structure. This is the reason why it is termed a hierarchical codebook.

For example, Figure 2 shows the case with $n_{c}=3$ and $n_{f}=2$. The partition of the channel sample space consists of $N_{c}=8$ coarse encoding regions, the $i_{c}$ th of which is denoted as $\mathcal{R}_{i_{c}}$, as a result of the mLBG VQ procedure. Each individual coarse encoding region is again decomposed into $N_{f}=4$ fine encoding regions, the $i_{f}$ th of which is $\mathcal{R}_{i_{c}, i_{f}}$ in case that it is based on $\mathcal{R}_{i_{c}}$. In the end, we get $N_{c b}=32$ fine codes associated with the corresponding fine encoding regions.

\subsection{Operation scenario}

The operation scenario of the hierarchical codebook deployment, which is also termed a combined codebook in this article, is as follows.

(i) Coarse feedback: feedback of the codebook index $i_{c}$. For every coarse feedback period $\tau_{c}$, the MS sends the $n_{c}$ bit codebook index $i_{c}$ back to the associated BSs so as to indicate the chosen codebook. Based on the channel information observed for the time period $\tau_{c}$, the MS quantizes the channel matrix and finds the best code in terms of the chordal distance. The index of the chosen code $\mathbf{C}_{\left(i_{c}-1\right) N_{f}+i_{f}} \in \mathbb{C}^{N_{t t} \times N_{r}}$ can be decomposed into two parts, for example, the codebook index part $i_{c}$ and the code index part $i_{f}$. The coarse feedback involves sending back $i_{c}$.

(ii) Fine feedback: feedback of the code index $i_{f}$.

For every fine feedback period $\tau_{f}$ within $\tau_{c}$, the MS sends the $n_{f}$ bit code index $i_{f}$ back to the associated BSs so as to indicate the chosen code. It means that the MS performs the channel quantization for every $\tau_{f}$, but the scope of candidate codes is restricted within the codebook $\mathcal{C}_{i_{c}}^{c}$. This can save a lot of computational burden for the MS, since the number of candidate codes is $N_{f}$ instead of $N_{c} N_{f}$. The fine feedback involves sending back $i_{f}$.

The BSs collect the coarse and fine feedback messages, and combine this information to find the chosen code, which is one of $N_{c b}=2^{n_{c}+n_{f}}$ fine codes which are predefined and shared by both BSs and MSs.

There are several points to be worth our attention.

(1) The feedback periods $\tau_{c}$ and $\tau_{f}$ have a significant effect on the system performance. It is a challenging task to find an analytical solution for calculating an optimum feedback period. The optimization problem is supposed to maximize the performance or to minimize the performance degradation compared with the ideal case $\left(\tau_{c}, \tau_{f}\right.$ are equal to the shortest possible feedback period), and it is a function not only of the dimension of the channel matrix to quantize, the speed of the MS, and the carrier frequency, but also of the number of feedback bits $n_{c}, n_{f}$ which decide the resolution of the channel quantizer.

(2) Once found, $\tau_{c}$ and $\tau_{f}$ can have fixed values for given channel-related parameters (the channel matrix dimension, the carrier frequency, and the speed of 


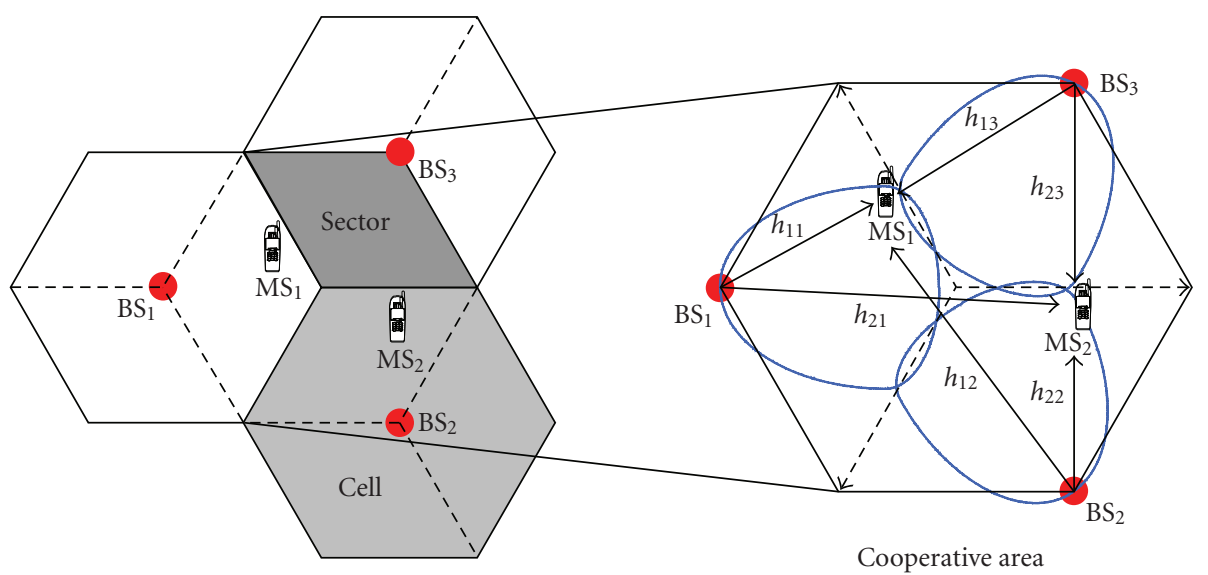

FIgURE 3: CA topology based on 3-sector-cell system.

the MS) and the number of feedback bits, and are still able to guarantee the target performance. Therefore, we do not need a feedback period of variable length for given circumstances, which makes the system design problem easy. If parameters other than the mobile speed remain same, we only need to scale the feedback period with respect to the mobile speed.

(3) Within the coarse feedback period $\tau_{c}$, the resulting codebook has a limited scope, since it selects the best code from the chosen codebook only. If the actual channel realization falls into a different coarse encoding region, the quantization error would be significant.

The BS may benefit from saving the coarse codebook $C^{c}=\left\{\mathbf{C}_{1}^{c}, \mathbf{C}_{2}^{c}, \ldots, \mathbf{C}_{N_{c}}^{c}\right\}$ into memory, just in case that MSs are temporarily disabled to send fine feedback messages to BSs. In this case, the BSs are accessible to coarse feedback indices only. However, the BSs can still reconstruct the channel if the coarse codebook is available at the BSs. The BS is supposed to have two codebooks, one of which is the coarse codebook of size $N_{c}$, and another is the combined codebook of size $N_{c b}$. Only the combined codebook needs to be saved on the MS side.

\subsection{Further comments}

The feedback overhead reduction of the combined codebook can be also achieved by constructing a single fine codebook followed by an adequate codeword assignment. For example, we can accomplish the same effect by assigning codewords to quantization regions in such a way that quantization regions close in chordal distance are assigned to codewords close, say, in Hamming distance. In addition, another alternative way to exploit temporal correlations to reduce the amount of feedback is by differential encoding, that is, by transmitting only the difference between the actual index and the previous index. If the channel has slightly changed, only the least significant bits will be transmitted. The most significant bits will be transmitted only when the channel has experienced an abrupt change.
On the other hand, the hierarchical codebook design method can be further improved by endowing tracking capability. A subspace tracking codebook can be defined as a subset of the entire codebook which consists of neighboring codewords of the currently chosen codeword. As this small size neighboring codebook is able to change its elements adaptively to the current status, it is capable of tracking a subspace, which leads to a further reduction of the feedback overhead.

\section{NUMERICAL RESULTS}

In this section, we present numerical results. First, simulations have been performed for the $2 \mathrm{BSs}-2 \mathrm{MSs}$ and $3 \mathrm{BSs}-$ 2MSs cases to evaluate the performance of the proposed channel quantizer and the codebook construction method. Two (three) BSs are cooperating to transmit data signal for two MSs through the same resources at the same time. Both BSs and MSs have a single antenna, so it yields $2 \times 2$ and $2 \times 3$ overall channel matrices, respectively. We employ the transmit zero-forcing filter as an example ofbeamforming scheme to prove the quality of the proposed quantization method. The extended 3GPP spatial channel model (SCM) is used for the simulations; and the proposed methods are tested for an urban macro channel with a mobile speed of $10 \mathrm{~m} / \mathrm{s}$. (The MATLAB code provided in [25] supports a channel matrix generation function for links between multiple BSs and multiple MSs.) The system performance is evaluated in terms of the received SINR at the MS. Simulations are performed for 30,000 channel realizations and the cumulative distribution function (CDF) at one MS is obtained. OFDMA is assumed as the data transmission scheme and we focus on one subcarrier. The transmit power at the $\mathrm{BS}$ is set to be $10 \mathrm{~W}$ and it is equally allocated to 1201 subcarriers.

The cooperative area (CA) topology is shown in Figure 3. As in the conventional cellular topology, one cell that is composed of three sectors and the hexagonal area, which is composed of three sectors which are served by three BSs, forms a CA. Two MSs in the CA are served by three BSs simultaneously. In case of the $2 \mathrm{BS}$-2MSs case, two BSs which maintain 
the strongest two links with MSs are chosen for downlink transmission. The cell radius is $600 \mathrm{~m}$ and MSs are equally distributed in the CA for every drop.

The transmit zero-forcing filter formula follows (3), based on downlink channel information which is either perfect channel ( $\mathrm{pCh})$, or is provided by a downlink channel estimation method which is shared by the BSs through a prompt, error free backbone network (centralized CA denoted by cCA), or is acquired by the analog pilot retransmission method (distributed CA denoted by dCA), or is captured and reconstructed by looking up an $n$ bit codebook ( $n$ bit channel quantization referred to as $n \mathrm{bCQ}$ ). The cCA case assumes that the system employs a time division duplex (TDD) scheme and the backbone network connecting associated BSs is delay free and error free. The downlink channel state information can be acquired by estimating uplink channel by using uplink-downlink channel reciprocity, when there exists a direct link between a BS and an MS. In simulations, the uplink channel is assumed to be estimated by using the uplink pilot signal. The nondirect link channel information can be provided by BSs with direct links through prompt data communication over the backbone network. In the analog pilot retransmission method, the MS sends the received pilot which pertains to the downlink channel state information to all associated BSs over the uplink channel $[1,6]$. In this case, two pilots are required in the uplink. One is for conveying the received pilot directly to the BSs (analog pilot retransmission), and the other is for estimating the uplink channel itself, which is necessary to compensate the retransmitted pilot for the uplink channel influence so as to acquire the downlink channel information. Therefore, these two pilots should be adjacent in time and frequency. Since the estimated version of the channel state information is used to peel off distortions caused by the uplink channel from the retransmitted pilot, this method is vulnerable to noise enhancement effects.

The BSs are assumed to be aware of the large-scale fading of the channel; and the channel quantization process (9) is based on true channel information. (Some readers may find a direct comparison between cCA and $n \mathrm{bCQ}$ inadequate in the sense that cCA is based on the realistic channel estimation method while $n b C Q$ is based on the ideal channel knowledge. However, the performance of the cCA case is provided here as a mere reference for the mapping of the performance of the proposed method in relation to an alternative method.) The codebooks are acquired by the modified LBG VQ algorithm. The feedback link is error free and delay free.

First, two proposals concerning the channel quantization model are evaluated. One model $\left(\hat{\mathbf{H}}_{j}^{d}\right.$, equation $\left.(9)\right)$ adopts the channel directional information only, and the other $\left(\hat{\mathbf{H}}_{j}\right.$, equation (8)) takes the channel magnitude information into account, as well as the channel directional information. Figure 4 shows the CDF of the SINR for the 3BSs-2MSs case. The channel directional information-based model (4bCDI, 5bCDI) performs closely to or in the low SINR region even slightly better than the model which combines the directional and magnitude information (4bCDMI, 5bCDMI). We assume that the BSs have access to the link strengths (large-scale fading due to path loss and shadowing)

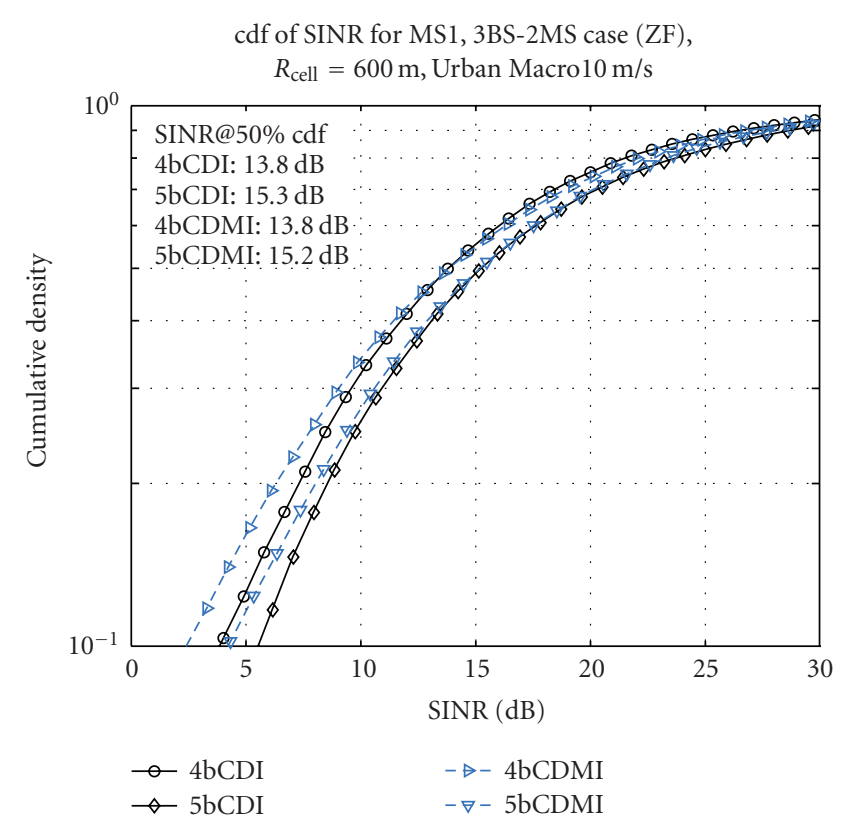

FIGURE 4: Performance comparison of channel quantization models (4bCDI: 4-bit-channel directional information-based quantization; 5bCDI: 5-bit CDI; 4bCDMI: 4-bit channel directional/magnitude information-based quantization; 5bCDMI: 5-bit CDMI).

for both cases, and the BSs have perfect knowledge of $\boldsymbol{\Sigma}_{j}^{(S)}$ for the latter case. Simulation results indicate that the extra channel magnitude information does not improve the SINR performance in these cases. (We should be careful in interpreting the simulation results. We have simulated relatively low-bit (4 and 5 bits) quantization cases. In this case, the precision of the channel directional information is more relevant to the system performance than the channel magnitude information. The channel magnitude information can play an important role for higher-bit quantization cases, where the accuracy of the channel directional information is sufficiently high that only the magnitude information can help improve performance. In this paper, we focus on the limited feedback case in which the channel directional information matters most.) Please note that in this paper the channel quantizer (CQ) or the subspace-based CQ refers to the channel directional information-based model, unless otherwise mentioned.

Figure 5 shows the CDF of the SINR for the 2BSs-2MSs case. At 50\% outage SINR, the 3-bit channel quantizer (3bCQ) shows 7.8 dB gain over the analog pilot retransmission case (dCA) and it is only $0.1 \mathrm{~dB}$ away from the centralized CA (cCA). The channel matrix at MS $j, \mathbf{H}_{j}(j=1,2)$, is in this case a $2 \times 1$ complex vector and this is represented by a codebook of size $2^{3}=8$. Compared with the channel quantization method, the resource efficient dCA case requires 3pilot tones per MS in case of FDD. Therefore, the proposed scheme performs much better than the pilot retransmission method without requiring extra resources. Figure 6 deals with simulation results of the $3 \mathrm{BSs}-2 \mathrm{MSs}$ case. The $3 \mathrm{bCQ}$, $4 \mathrm{bCQ}$, and $5 \mathrm{bCQ}$ cases have $3.2 \mathrm{~dB}, 5.0 \mathrm{~dB}$, and $6.5 \mathrm{~dB}$ gains over the dCA case, respectively. In this case, the proposed 


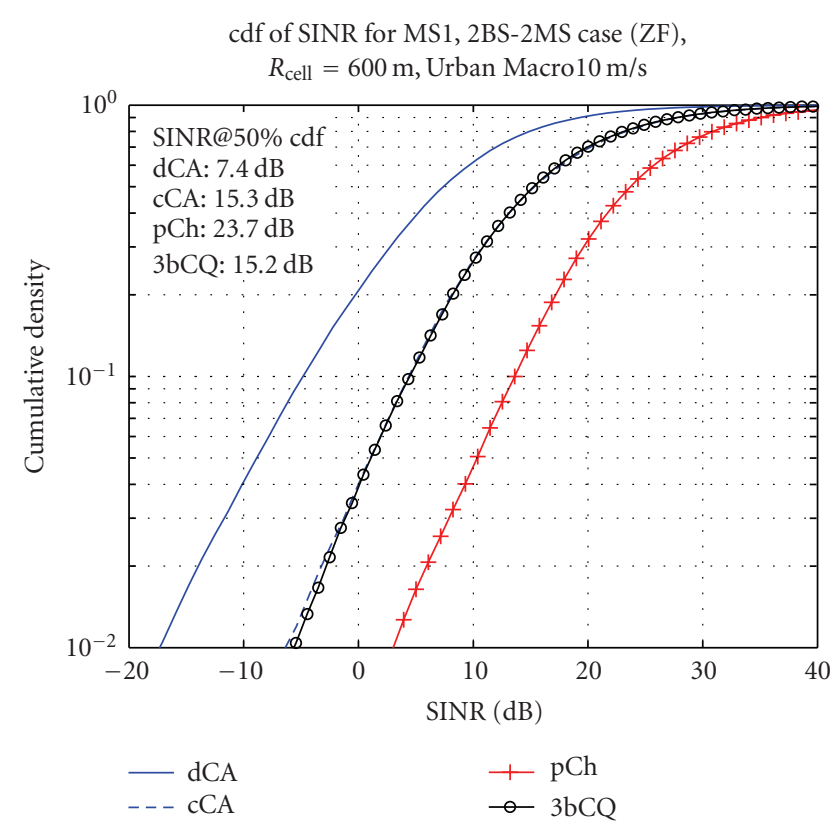

FIGURE 5: 2BSs-2MSs case simulation results (dCA: distributed CA; cCA: centralized CA; pCh: perfect channel; 3bCQ: 3-bit CQ).

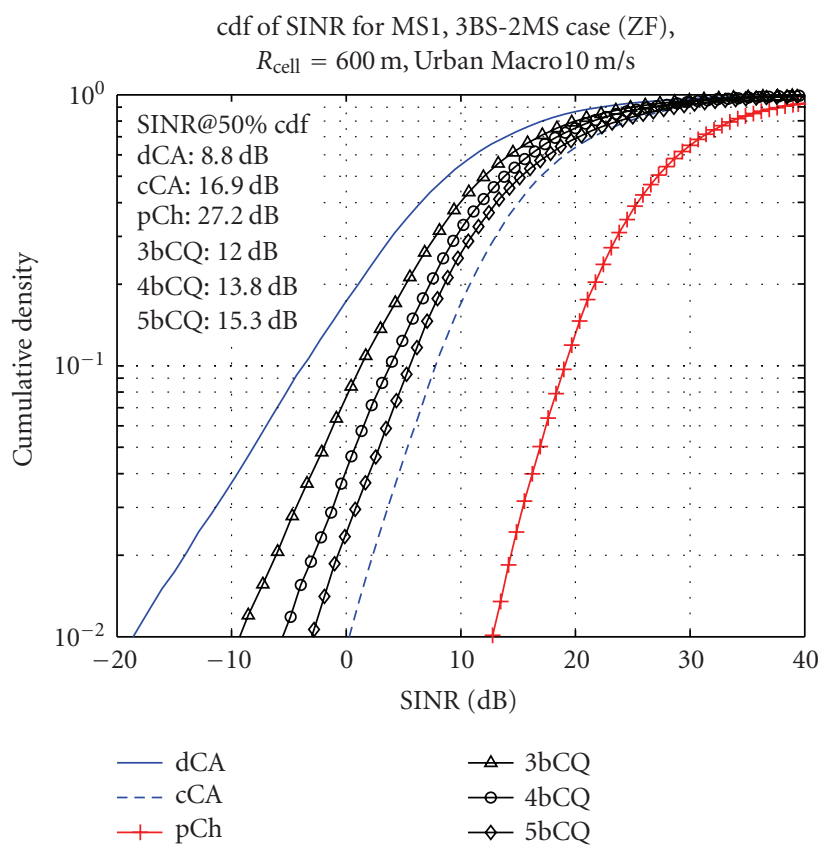

FIGURE 6: 3BSs-2MSs case simulation results (dCA: distributed CA; cCA: centralized CA; pCh: perfect channel; 3bCQ: 3-bit CQ; 4bCQ: 4 bit CQ; 5bCQ: 5-bit CQ).

method still has a lot of room for improvement even though the expected gain over the conventional method is not insignificant. The gap between the proposed method and the ideal case can be reduced by adopting a smart scheduling strategy like user grouping which selects users with orthogonal channel signatures so as to reduce interferences between different users [18].
The proposed method is to quantize the channel matrix based on the chordal distance, and the LBG VQ algorithm is modified as such. Conventional VQ methods use the Euclidean distance instead. Is the subspace-based method better than the conventional method? A performance comparison result is shown in Figure 7. The Euclidean distance-based CQ ( $n$ beCQ) adopts the Euclidean distance as a distance metric for channel quantization. The simulation results show that the subspace-based CQ has a substantial gain over the Euclidean distance-based CQ. At 50\% outage SINR, the 4 bCQ and 5bCQ outperform the 4 beCQ and 5beCQ by $2.9 \mathrm{~dB}$ and $3.0 \mathrm{~dB}$, respectively.

There exists another CQ method which exploits Givens rotations [26]. This method allows us to represent the column space basis vectors $\mathbf{U}^{(S)} \in \mathbb{C}^{t \times n}$ of the channel by $(2 t-1) n-n^{2}$ real numbers. $t=3, n=1$ holds for the 3BSs$2 \mathrm{MSs}$ case, and it requires 4 real number parameters $\left(\phi_{1,2}\right.$, $\left.\phi_{1,3}, \theta_{1,1}, \theta_{1,2}\right)$ for channel matrix construction. The performance comparison result between the proposed method and the Givens-rotation-based channel matrix decomposition method is shown in Figure 8. The Givens-rotation-based method with $n$-bit feedback is denoted by $n$ bGR. 4 bGR allocates 1 bit for each parameter, and 5bGR assigns 2, 2, 1, and 0 $\operatorname{bit}(\mathrm{s})$ for $\phi_{1,2}, \phi_{1,3}, \theta_{1,1}$, and $\theta_{1,2}$, respectively. (In this case, the value of $\theta_{1,2}$ is predefined and fixed.) At $50 \%$ outage SINR, the $4 \mathrm{bCQ}$ case outperforms the $4 \mathrm{bGR}$ case by $2.5 \mathrm{~dB}$, while the $5 \mathrm{bCQ}$ case shows comparable performance to the 5bGR case of which the computational complexity at MS is higher than that of the 5bCQ case.

The performance of the combined codebook is shown in Figure 9. Simulations have been performed for the 2BSs$2 \mathrm{MSs}$ case. The combined codebooks are acquired by the modified LBG VQ algorithm and the hierarchical codebook construction method as described in Section 5. The combined codebook of the $n_{c}$-bit coarse and $n_{f}$-bit fine feedbacks with corresponding feedback periods $\tau_{c}$ and $\tau_{f}$ is denoted by $n_{c}+n_{f}$ bCQ $\left(\left[\tau_{c}\right],\left[\tau_{f}\right]\right)$, where the unit of feedback period is the number of OFDM symbols: $[\tau]=m$, when $\tau=m \cdot T_{s}\left(T_{s}=71.37 \mu s\right)$. At $50 \%$ outage SINR, the 5 -bit codebook case (5bCQ), which is generated by the hierarchical codebook construction method, shows $11.8 \mathrm{~dB}$ and $3.9 \mathrm{~dB}$ gains over the analog pilot retransmission case (dCA) and the centralized CA case (cCA), respectively. In this case, the 5-bit feedback is being sent back for every symbol. The 3 +2 -bit combined codebook with the empirically found optimum feedback period pair $\left(\left[\tau_{c}\right],\left[\tau_{f}\right]\right)=(10,5),\left(3+2 \mathrm{bCQ}_{1}\right)$ is less than $0.1 \mathrm{~dB}$ away from the performance of the $5 \mathrm{bCQ}$ case, even though some degradation is observed in the low SINR region. In terms of the required resources, the 5bCQ case requires 5 bits/symbol for feedback, while the $3+2 b_{C Q}$ case needs only $0.7 \mathrm{bit} / \mathrm{symbol}$. Thus, the $3+2$-bit combined codebook can achieve the performance of the 5-bit codebook with negligible degradation by using just $14 \%$ of the feedback resource.

We have tested the $3+2$ bCQ case with various suboptimum feedback periods, for example, $(10,10)$ and $(20,5)$, and each case is denoted by the subscripts 2 and 3, respectively. None of these cases outperforms the optimum case $(10,5)$, and performance degradations are $0.7 \mathrm{~dB}$ and $0.8 \mathrm{~dB}$ for the 


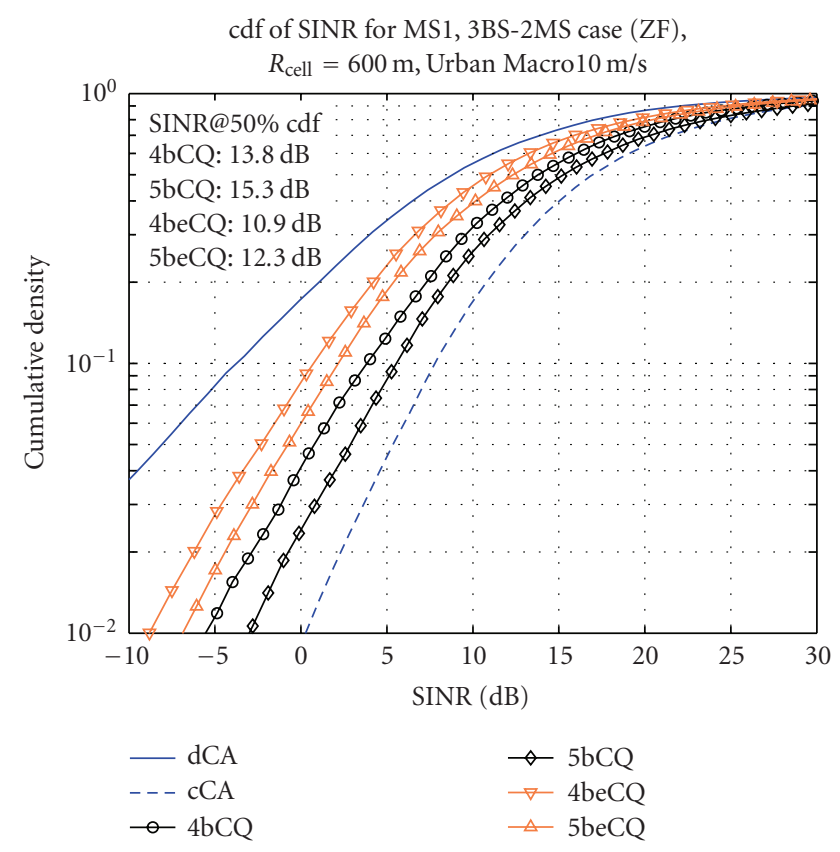

FIgURE 7: Performance comparison of the Euclidean distance-based CQ and the subspace-based CQ (dCA: distributed CA; cCA: centralized CA; 4bCQ: 4 bit subspace-based CQ; 5bCQ: 5-bit subspacebased CQ; 4beCQ: 4-bit Euclidean distance-based CQ; 5beCQ: 5-bit Euclidean distance-based CQ).

longer fine feedback period case and the longer coarse feedback period case, respectively. The following points should be noted with respect to simulation results.

\section{(i) The optimum feedback period pair guarantees the target performance}

In this case, $\left(\left[\tau_{c}\right],\left[\tau_{f}\right]\right)=(10,5)$ is the optimum feedback period pair, and any other case with longer period results in performance degradation. The task of finding an optimum feedback period pair in an analytical way is an interesting topic for future research.

\section{(ii) The coarse feedback period is decisive in the performance}

Even though both $\tau_{c}$ and $\tau_{f}$ are important in deciding the performance, $\tau_{c}$ has a more profound impact than $\tau_{f}$, since the coarse codebook is associated with the bigger encoding region which entails a bigger error if the feedback information is outdated. Both the $(10,10)$ and $(20,5)$ cases show degradations in performance. The degradation of the $(20,5)$ case with a longer coarse feedback period is $0.1 \mathrm{~dB}$ which is bigger than the $(10,10)$ case with a longer fine feedback period, even though the former requires $0.55 \mathrm{bit} / \mathrm{symbol}$ feedback overhead while the latter needs $0.5 \mathrm{bit} / \mathrm{symbol}$ overhead. In short, the $(20,5)$ case performs worse than the $(10,10)$ case despite its higher feedback overhead, and this performance degradation comes from the suboptimum coarse feedback period.

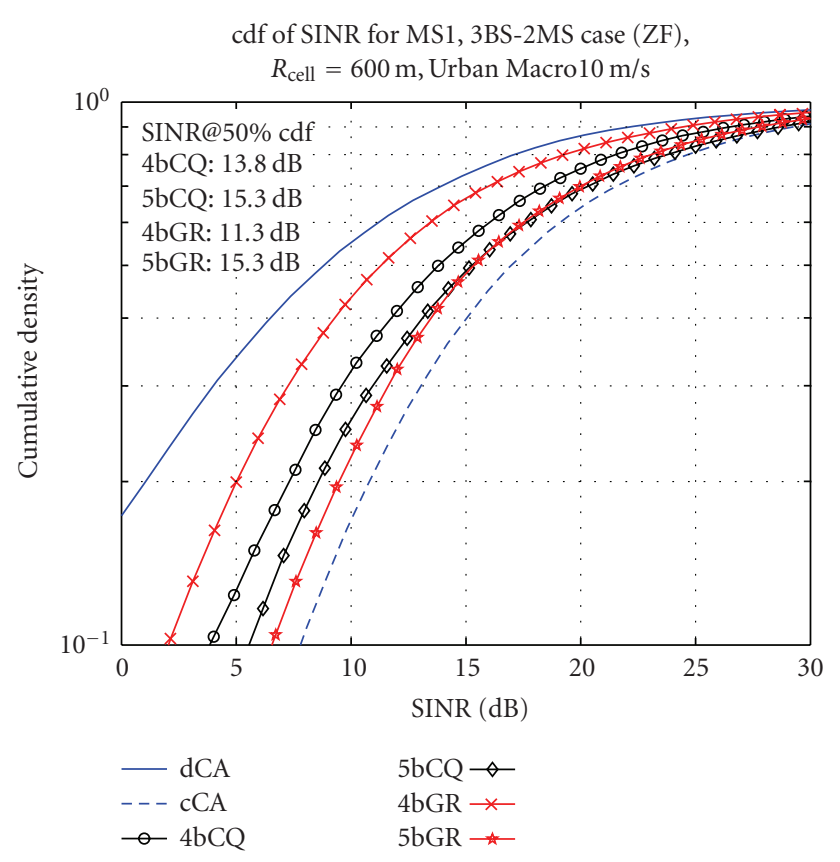

FIGURE 8: Performance comparison of the Givens rotation-based CQ and the subspace-based CQ (dCA: distributed CA; cCA: centralized CA; 4bCQ: 4-bit subspace-based CA; 5bCQ: 5-bit subspacebased CQ; 4bGR: 4-bit Givens-rotation-based CQ; 5bGR: 5-bit Givens-rotation-based CQ).

\section{CONCLUSIONS}

In this paper, we have investigated precoded MU-MIMO systems with limited feedback. The subspace-based channel quantization method is proposed as a way of providing BSs with downlink channel state information in the presence of interuser interference, which is applicable to the distributed COOPA systems as well as MU-MIMO systems. The subspace-based channel quantizer improves the system performance significantly, compared to the analog pilot retransmission method with relatively small feedback overhead. We also developed an efficient codebook construction algorithm based on well-known LBG VQ by adopting the chordal distance and modifying the optimality criteria accordingly. The codebooks generated by the proposed algorithm have better distance properties than Grassmannian codebooks that are currently available.

We have also proposed a feedback overhead reduction scheme which makes use of the temporal correlation of the channel. It constructs the codebook with a hierarchical structure so that the feedback index can be divided into two parts, that is, a coarse feedback which points to the codebook index and a fine feedback which indicates the code index. The resulting codebook is termed the combined codebook. The simulation results suggest that we can save a significant amount of feedback resources while maintaining the same performance level as the case with fully loaded feedback. The decision of the optimum feedback periods is an open issue for future research. 


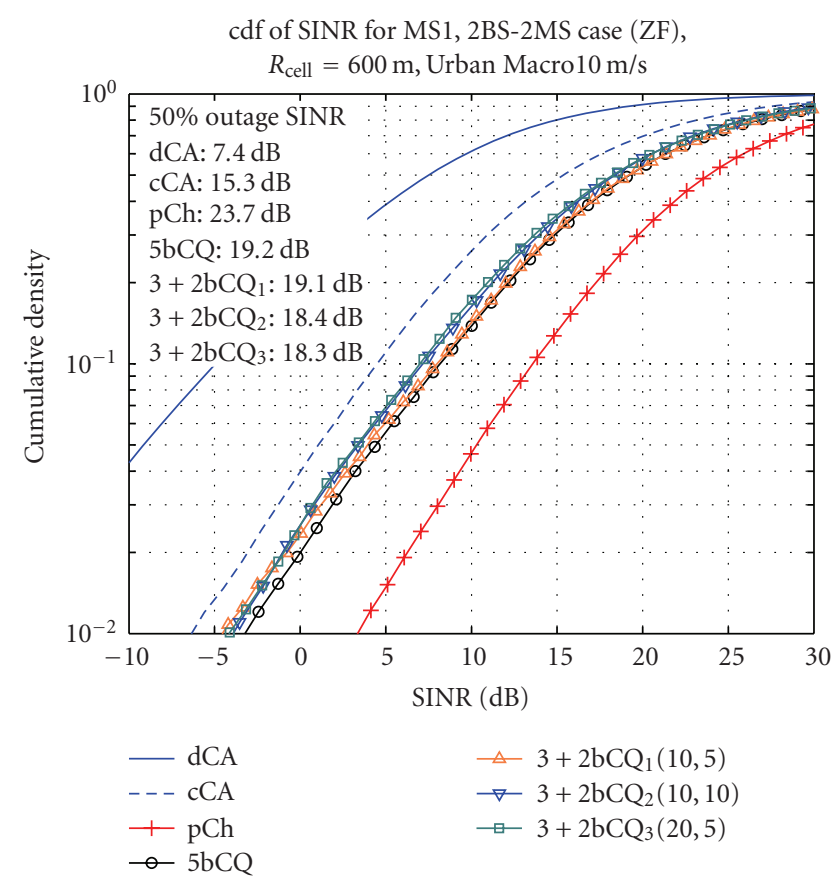

Figure 9: Combined codebook simulation results for $\left(n_{c}+n_{f} \mathrm{~b}\right)=$ $(3+2 b)$ case (dCA: distributed CA; cCA: centralized CA; pCh: perfect channel; 5bCQ: 5-bit CQ; $3+2 \mathrm{bCQ}\left(\left[\tau_{c}\right],\left[\tau_{f}\right]\right)$ : 3-bit coarse and 2-bit fine feedbacks with a feedback period pair $\left(\left[\tau_{c}\right],\left[\tau_{f}\right]\right) \in$ $\{(10,5),(10,10),(20,5)\})$.

\section{APPENDIX}

\section{PROOF OF EQUATION (12)}

First, we provide the formula which explains how the chordal distance is related with the inner product, when it is used for two unit norm vectors $\mathbf{v}_{i}, \mathbf{v}_{j}$, where $\mathbf{v}_{i}^{\mathcal{H}} \mathbf{v}_{i}=\mathbf{v}_{j}^{\mathcal{H}} \mathbf{v}_{j}=1$;

$$
\begin{aligned}
d_{c}^{2}\left(\mathbf{v}_{i}, \mathbf{v}_{j}\right) & =\left(\frac{1}{\sqrt{2}}\left\|\mathbf{v}_{i} \mathbf{v}_{i}^{\mathcal{H}}-\mathbf{v}_{j} \mathbf{v}_{j}^{\mathcal{H}}\right\|_{F}\right)^{2} \\
& =\frac{1}{2} \operatorname{tr}\left(\left(\mathbf{v}_{i} \mathbf{v}_{i}^{\mathcal{H}}-\mathbf{v}_{j} \mathbf{v}_{j}^{\mathscr{H}}\right)\left(\mathbf{v}_{i} \mathbf{v}_{i}^{\mathcal{H}}-\mathbf{v}_{j} \mathbf{v}_{j}^{\mathscr{H}}\right)^{\mathscr{H}}\right) \\
& =\frac{1}{2} \operatorname{tr}\left(\mathbf{v}_{j}^{\mathscr{H}}\left(\mathbf{v}_{i} \mathbf{v}_{i}^{\mathcal{H}}-\mathbf{v}_{j} \mathbf{v}_{j}^{\mathcal{H}}\right)\left(\mathbf{v}_{i} \mathbf{v}_{i}^{\mathcal{H}}-\mathbf{v}_{j} \mathbf{v}_{j}^{\mathscr{H}}\right)^{\mathscr{H}} \mathbf{v}_{j}\right) \\
& =\frac{1}{2}\left(1-\mathbf{v}_{j}^{\mathscr{H}} \mathbf{v}_{i} \mathbf{v}_{i}^{\mathcal{H}} \mathbf{v}_{j}\right) \\
& =\frac{1}{2}\left(1-\left|\left\langle\mathbf{v}_{i}, \mathbf{v}_{j}\right\rangle\right|^{2}\right),
\end{aligned}
$$

where $\left\langle\mathbf{v}_{i}, \mathbf{v}_{j}\right\rangle=\mathbf{v}_{i}^{\mathscr{H}} \mathbf{v}_{j}$. The following property is used from the first to the second line: $\|\mathbf{A}\|_{F}=\sqrt{\operatorname{tr}\left(\mathbf{A} \mathbf{A}^{\mathscr{H}}\right)}$; and from the third to the fourth line, the $\operatorname{tr}(\cdot)$ operation is omitted since its argument has a scalar value. From (A.1), the decision criterion in terms of the chordal distance can be formulated as follows:

$$
\arg \min d_{c}\left(\mathbf{v}_{i}, \mathbf{v}_{j}\right)=\arg \max \left|\left\langle\mathbf{v}_{i}, \mathbf{v}_{j}\right\rangle\right|
$$

On the other hand, the column space basis vector $\mathbf{u}_{\mathrm{S}} \in$ $\mathbb{C}^{N_{t t} \times 1}$ of the channel vector $\mathbf{h} \in \mathbb{C}^{N_{t t} \times 1}$ can be found as follows (the user index $j$ is omitted for brevity):

$$
\mathbf{h}=\mathbf{U}_{h} \boldsymbol{\Sigma}_{h} \mathbf{V}_{h}^{\mathcal{H}}=v_{h} \sigma_{h} \mathbf{u}_{\mathrm{S}},
$$

where $v_{h}$ and $\sigma_{h}$ have scalar values and $\mathbf{u}_{S} \in \mathbb{C}^{N_{t t} \times 1}$. The rightmost form is an "economy size" version of the SVD, where $v_{h}$ is in effect the same as $\mathbf{V}_{h}$ (size: $\left.1 \times 1\right)$, and $\sigma_{h}$ is the only nonzero singular value in $\boldsymbol{\Sigma}_{h}$. The followings holds: $\sigma_{h}=\|\mathbf{h}\|_{2}$ and $v_{h} \in\{+1,-1\}$, since $\mathbf{h}$ is a vector. Therefore, $\mathbf{u}_{\mathrm{S}}$ can be expressed in terms of the directional vector of the channel as follows:

$$
\mathbf{u}_{\mathrm{S}}=v_{h} \frac{\mathbf{h}}{\|\mathbf{h}\|_{2}}=v_{h} \mathbf{v},
$$

where $\mathbf{v}=\mathbf{h} /\|\mathbf{h}\|_{2}$ is the directional vector of the channel. Since $v_{h}$ decides the sign only, the following criterion holds:

$$
\widehat{\boldsymbol{h}}=\arg \min _{\mathbf{c}_{i} \in \mathcal{C}} d_{c}\left(\mathbf{u}_{S}, \mathbf{c}_{i}\right)=\arg \max _{\mathbf{c}_{i} \in \mathcal{C}}\left|\left\langle\mathbf{v}, \mathbf{c}_{i}\right\rangle\right| .
$$

Therefore, the chordal distance-based channel quantization criterion (9) can be simplified to the inner product-based criterion (12).

\section{ACKNOWLEDGMENT}

Part of this work was presented at the International ITG/IEEE Workshop on Smart Antennas (WSA'07), Vienna, Austria, February 2007.

\section{REFERENCES}

[1] W. Zirwas, J. H. Kim, V. Jungnickel, et al., "Distributed organization of cooperative antenna systems," in Distributed Antenna Systems: Open Architecture for Future Wireless Communications, H. Hu, Y. Zhang, and J. Luo, Eds., chapter 10, Auerbach Publications, Boca Raton, Fla, USA, June 2007.

[2] M. K. Karakayali, G. J. Foschini, and R. A. Valenzuela, "Network coordination for spectrally efficient communications in cellular systems," IEEE Wireless Communications, vol. 13, no. 4, pp. 56-61, 2006.

[3] M. K. Karakayali, G. J. Foschini, R. A. Valenzuela, and R. D. Yates, "On the maximum common rate achievable in a coordinated network," in Proceedings of the IEEE International Conference on Communications (ICC '06), vol. 9, pp. 4333-4338, Istanbul, Turkey, June 2006.

[4] G. J. Foschini, K. Karakayali, and R. A. Valenzuela, "Coordinating multiple antenna cellular networks to achieve enormous spectral efficiency," IEE Proceedings Communications, vol. 153, pp. 548-555, 2006.

[5] G. J. Foschini, H. C. Huang, M. K. Karakayali, R. A. Valenzuela, and S. Venkatesan, "The value of coherent base station coordination," in Proceedings of the 39th Annual Conference on Information Sciences and Systems (CISS '05), The Johns Hopkins University, Baltimore, Md, USA, March 2005.

[6] W. Zirwas, E. Schulz, J. H. Kim, V. Jungnickel, and M. Schubert, "Distributed organization of cooperative antenna systems," in Proceedings of 12th European Wireless Conference, Athens, Greece, April 2006. 
[7] D. J. Love, R. W. Heath Jr., and T. Strohmer, "Grassmannian beamforming for multiple-input multiple-output wireless systems," IEEE Transactions on Information Theory, vol. 49, no. 10, pp. 2735-2747, 2003.

[8] B. M. Hochwald, T. L. Marzetta, T. J. Richardson, W. Sweldens, and R. Urbanke, "Systematic design of unitary space-time constellations," IEEE Transactions on Information Theory, vol. 46, no. 6, pp. 1962-1973, 2000.

[9] J. C. Roh and B. D. Rao, "Vector quantization techniques for multiple-antenna channel information feedback," in Proceedings of the International Conference on Signal Processing and Communications (SPCOM '04), pp. 402-406, Banalore, India, December 2004.

[10] J. C. Roh and B. D. Rao, "Design and analysis of MIMO spatial multiplexing systems with quantized feedback," IEEE Transactions on Signal Processing, vol. 54, no. 8, pp. 2874-2886, 2006.

[11] D. J. Love, R. W. Heath Jr., W. Santipach, and M. L. Honig, "What is the value of limited feedback for MIMO channels?" IEEE Communications Magazine, vol. 42, no. 10, pp. 54-59, 2004.

[12] N. Jindal, "Finite rate feedback MIMO broadcast channels," in Proceedings of the Workshop on Information Theory and Its Applications (ITA '06), San Diego, Calif, USA, February 2006.

[13] J. A. Nossek, M. Joham, and W. Utschick, "Transmit processing in MIMO wireless systems," in Proceedings of the 6th IEEE Circuits and Systems Symposium on Emerging Technologies: Frontiers of Mobile and Wireless Communication (CASSET '04), vol. 1, pp. 18-23, Shanghai, China, May-June 2004.

[14] M. Tomlinson, "New automatic equaliser employing modulo arithmetic," Electronics Letters, vol. 7, no. 5-6, pp. 138-139, 1971.

[15] H. Harashima and H. Miyakawa, "Matched-transmission technique for channels with intersymbol interference," IEEE Transactions on Communications, vol. 20, no. 4, pp. 774-780, 1972.

[16] Q. H. Spencer, A. L. Swindlehurst, and M. Haardt, "Zeroforcing methods for downlink spatial multiplexing in multiuser MIMO channels," IEEE Transactions on Signal Processing, vol. 52, no. 2, pp. 461-471, 2004.

[17] V. Stankovic and M. Haardt, "Multi-user MIMO downlink precoding for users with multiple antennas," in Proceedings of the 12th Meeting of the Wireless World Research Forum (WWRF '04), Toronto, Ontario, Canada, November 2004.

[18] T. Yoo and A. Goldsmith, "On the optimality of multiantenna broadcast scheduling using zero-forcing beamforming," IEEE Journal on Selected Areas in Communications, vol. 24, no. 3, pp. 528-541, 2006.

[19] D. J. Love and R. W. Heath Jr., "Limited feedback unitary precoding for spatial multiplexing systems," IEEE Transactions on Information Theory, vol. 51, no. 8, pp. 2967-2976, 2005.

[20] S. Zhou and B. Li, "BER criterion and codebook construction for finite-rate precoded spatial multiplexing with linear receivers," IEEE Transactions on Signal Processing, vol. 54, no. 5, pp. 1653-1665, 2006.

[21] T. Yoo, N. Jindal, and A. Goldsmith, "Finite-rate feedback MIMO broadcast channels with a large number of users," in Proceedings of the IEEE International Symposium on Information Theory (ISIT'06), pp. 1214-1218, Seattle, Wash, USA, July 2006.

[22] Y. Linde, A. Buzo, and R. M. Gray, "An algorithm for vector quantizer design," IEEE Transactions on Communications, pp. 702-710, 1980.

[23] D. J. Love, "Personal webpage on Grassmannian subspace packing," http://cobweb.ecn.purdue.edu/djlove/grass.html.
[24] K. Huang, B. Mondal, R. W. Heath Jr., and J. G. Andrews, "Limited feedback for temporally-correlated channels: feedback rate and delay," to appear in IEEE Transactions on Information Theory, http://www.citebase.org/abstract?id=oai:arXiv .org:cs/0606022.

[25] D. S. Baum, J. Salo, M. Milojevic, P. Kyösti, and J. Hansen, "MATLAB implementation of the interim channel model for Beyond-3G systems (SCME)," http://www.tkk.fi/Units/ Radio/scm/.

[26] J. C. Roh and B. D. Rao, "An efficient feedback method for MIMO systems with slowly time-varying channels," in Proceedings of the IEEE Wireless Communications and Networking Conference (WCNC '04), vol. 2, pp. 760-764, Atlanta, Ga, USA, March 2004. 